\title{
A Kaleodoscopic View of Fuzzy Stochastic Optimization*
}

\author{
Yves Tinda Mangongo', Justin Dupar Busili Kampempe1, Monga Kalonda Luhandjula² \\ ${ }^{1}$ Département de Mathématiques et Informatique, Université de Kinshasa, Kinshasa, Democratic Republic of the Congo \\ ${ }^{2}$ Département de Mathématique-Informatique, Université de Kisangani, Kisangani, Democratic Republic of the Congo \\ Email: yves.mangongo@unikin.ac.cd, justin-dupar.kampempe@unikin.ac.cd,jp-monga@yahoo.com
}

How to cite this paper: Mangongo, Y.T., Kampempe, J.D.B. and Luhandjula, M.K. (2021) A Kaleodoscopic View of Fuzzy Stochastic Optimization. American Journal of Operations Research, 11, 283-308. https://doi.org/10.4236/ajor.2021.116018

Received: September 9, 2021

Accepted: October 30, 2021

Published: November 2, 2021

Copyright (c) 2021 by author(s) and Scientific Research Publishing Inc. This work is licensed under the Creative Commons Attribution International License (CC BY 4.0).

http://creativecommons.org/licenses/by/4.0/

\begin{abstract}
The last three decades have witnessed development of optimization under fuzziness and randomness also called Fuzzy Stochastic Optimization. The main objective of this new field is the need for basing many human decisions on information which is both fuzzily imprecise and probabilistically uncertain. Consistency indexes providing a union nexus between possibilities and probabilities of uncertain events exist in the literature. Nevertheless, there are no reliable transformations between them. This calls for new paradigms for coping with mathematical models involving both fuzziness and randomness. Fuzzy Stochastic Optimization (FSO) is an attempt to fulfill this need. In this paper, we present a panoramic view of Fuzzy Stochastic Optimization emphasizing the methodological aspects. The merits of existing methods are also briefly discussed along with some related theoretical aspects.
\end{abstract}

\section{Keywords}

Optimization, Randomness, Fuzziness, Fuzzy Random Variable

\section{Introduction}

Mathematical programming is a very useful tool in the hands of a decision maker. As a matter of fact, many decision problems including allocation of resources, transportation, affectation, production, etc. may be cost into an optimization framework. An interested reader may consult the paper by Dan [1], where a complex deterministic optimization problem is discussed. According to $\mathrm{Za}$ deh's incompatibility principle [2], when the complexity of a system increases, our aptitude to make precise statements on it decreases up to a threshold from

*Authors express their gratefulness feelings to an anonymous Referee, whose comments helped increase the quality of the paper. 
which precision and significance become mutually exclusive characteristics. In this connection, the noted philosopher Nietzche was quoted as saying: "No one is gifted with immaculate perception". The Physics Nobel Laureate Feynman shared this view when he pointed out: "When dealing with a mathematical model, special attention should be paid to imprecision in data". False certainty is bad science and it could be dangerous if it stunts articulation of critical choices. Fuzzy Optimization and Stochastic Programming [3] [4] provide a corpus of scientific knowledge that allows incorporating respectively fuzziness and randomness in an optimization context. Nevertheless the two kinds of imprecision may occur simultaneously in some optimization problems, hence, the interest of discussing ways for combining the two kinds of uncertainty in a mathematical programming framework. For some examples of problems involving both fuzzy quantities and random data, we invite the reader to consult [5] [6] [7]. The above mentioned approaches of getting rid of fuzziness and randomness at once in an optimization problem constitute the subject matter of this paper. First and foremost, we present existing mathematical formalisms for combining fuzziness and randomness (Section 2). Second, we discuss how these formalisms are used, with good reasons to deal with hybrid situations involving fuzziness and randomness in an optimization setting (Section 3). Some applications of these approaches are discussed in Section 4. We end up, in Section 5, with some concluding remarks along with some lines for further developments in this field. It is worth mentioning that instead of using a mere approximation approach for defuzifying data, one may use semi-infinite mathematical programming to have exact results. But this is a subject matter for another paper.

\section{Mathematical Formalisms for Combining Fuzziness and Randomness}

\subsection{Probability of a Fuzzy Event}

According to Zadeh [8], a probability of a fuzzy event is the expected value of its membership function. So given a fuzzy event $A$ on a probability space $\Omega$, its probability is defined as follows:

$$
P(A)=E\left(\mu_{A}\right)=\int_{\Omega} \mu_{A}(x) \mathrm{d} x .
$$

For details on probability of a fuzzy event, the interested reader is invited to consult [9].

\subsection{Probabilistic Set}

Given a set $X$ and a probability space $\Omega$, a probabilistic set $A$ on $X$ is a fuzzy set of $X \times \Omega$, whose membership function:

$$
\begin{aligned}
\mu_{A}: X \times \Omega & \rightarrow[0,1] \\
(x, \omega) & \longmapsto \mu_{A}(x, \omega)
\end{aligned}
$$

is measurable.

Intersections and unions of fuzzy probability sets are defined using T-norms 
or Conorms respectively. We refer the reader for properties of probabilistic sets to Hirota [10]. It is worth mentioning that probabilistic sets are appropriate tools for modelizing decisions in a fuzzy random environment.

\section{Uncertain Probabilities}

To put things in context, consider a continuous random variable $X$ with density function $f(x, \theta)$, where $\theta$ is a parameter describing the density function. If $\theta$ can be generated as a fuzzy number $\tilde{\theta}$, then $X$ has density $f(x, \tilde{\theta})$ and the probability of the event: " $X$ is between $c$ and $d$ " is a fuzzy set whose $\alpha$-cuts are defined as follows:

$$
[\tilde{P}(c \leq X \leq d)]^{\alpha}=\left\{\int_{c}^{d} f(x, \theta) \mathrm{d} x \mid \theta \in \tilde{\theta}^{\alpha} ; \int_{-\infty}^{\infty} f(x, \theta) \mathrm{d} x=1\right\} .
$$

The first two moments of $X$ are defined, through their $\alpha$-cuts as follows.

$$
\begin{gathered}
\tilde{m}_{X}^{\alpha}(\theta)=\left\{\int_{-\infty}^{\infty} x f(x, \theta) \mathrm{d} x \mid \theta \in \tilde{\theta}^{\alpha} ; \int_{-\infty}^{\infty} f(x, \theta) \mathrm{d} x=1\right\}, \\
\left(\tilde{\sigma}_{X}^{2}\right)^{\alpha}(\theta)=\left\{\int_{-\infty}^{\infty}\left(x-m_{X}(\theta)\right)^{2} f(x, \theta) \mathrm{d} x \mid \theta \in \tilde{\theta}^{\alpha} ; m_{X}(\theta) \in \tilde{m}_{X}^{\alpha}(\theta) ;\right. \\
\left.\int_{-\infty}^{\infty} f(x, \theta) \mathrm{d} x=1\right\} .
\end{gathered}
$$

Random variables $X_{i}, i=1,2, \cdots, n$ with fuzzy parameters and having joint density function $f\left(x_{1}, \cdots, x_{n}, \tilde{\theta}\right)$ and marginal density function $f_{i}\left(x_{i}, \tilde{\theta}\right)$ are said to be independent if, for $\alpha \in(0,1]$ and for all $\theta \in \tilde{\theta}^{\alpha}$,

$$
f\left(x_{1}, \cdots, x_{n}, \theta\right)=\prod_{i=1}^{n} f_{i}\left(x_{i}, \theta\right) .
$$

For readers interested in more details on uncertain probabilities, we recommend the authoritative scientific papers by Buckley and Eslami [11] [12].

\subsection{Fuzzy Random Variable}

A fuzzy random variable (frv) on a probability space $(\Omega, F, P)$ is a fuzzy-valued function:

$$
\begin{aligned}
X^{\alpha}: \Omega & \rightarrow F_{0}(\mathbb{R}) \\
\omega & \longmapsto X_{\omega}
\end{aligned}
$$

such that for every Borel set $A$ of $\mathbb{R}$ and for every $\alpha \in(0,1],\left(X^{\alpha}\right)^{-1}(A) \in F$. Here $F_{0}(\mathbb{R})$ and $X^{\alpha}$ stand for the set of fuzzy numbers and the set-valued function:

$$
\begin{aligned}
X^{\alpha}: \Omega & \rightarrow 2^{(\mathbb{R})} \\
\omega & \longmapsto X_{\omega}^{\alpha}=\left\{x \in \mathbb{R} \mid X_{\omega}(x) \geq \alpha\right\}
\end{aligned}
$$

respectively.

Zadeh's decomposition principle extends quite naturally to frvs. Moreover $X^{\alpha}(\alpha \in(0,1])$ are random intervals and frvs possesses the Radon-Nikodym property. A considerable body of literature has grown out of the concept of frv, 
in a wide range of fields (e.g. [13] [14]).

\subsection{Random Fuzzy Variable}

A random fuzzy variable is a map from a possibilistic set to a probabilistic one, verifying some measurability conditions [15]. An interested reader may consult [16] for details on random fuzzy variables.

\subsection{Other Approaches}

Other approaches to blending epistemic and aleatory forms of uncertainty include imprecise probabilities in the style of Peter Walley [17] and p-boxes defined by Ferson [18]. These approaches may be used to enrich models and methods for Fuzzy Stochastic Optimization.

\section{Fuzzy Stochastic Optimization Models}

\subsection{Preamble}

Fuzzy Stochastic Optimization (FSO) models are numerous and varied (e.g. [19]). They aim at stretching applicability of Optimization models to situations where both fuzziness and randomness are in the state of affairs. The back bone of FSO models is "the whole man doctrine" that urges us to bring everything we have to bear in our subject. Thwarting, suppressing or down playing uncertainty or imprecision in a mathematical model offers no other chance to that model but to churn out meaningless outcomes. This is in tune with the well-known Computer science rule: "Garbage in, Garbage out".

\subsection{Types of FSO Models}

In this paper, we consider 5 categories of FSO models. This classification is far form being exhaustive. It just reflects the main models encountered in the literature. The classification has been done according to the following criteria: the way fuzziness and randomness enter into the problem, the mathematical formalism used to account of involved hybrid uncertainty (see above), the structure of the optimization problem, the number of objective functions, the number of stages and the numbers of levels. The first class $\left(C_{1}\right)$ contains mathematical programming problems under fuzziness and randomness. Models on this class have one objective function and a limited number of constraints. Moreover the decision is taken here and now. For examples of models in category $C_{1}$, we refer the reader to papers [20] [21]. If we consider temporal and level dimensions, then we get: fuzzy stochastic version of Dynamic programming (category $C_{2}$ ) and multilevel optimization under randomness and fuzziness (category $C_{3}$ ).

Examples of models in Categories $C_{2}$ and $C_{3}$ may be found in references [22]. Another class of Fuzzy stochastic optimization models that came to the fore during the 1990s is the Fuzzy stochastic robust category $\left(C_{4}\right)$. Here, instead of specifying feasibility of an action by a set of inequalities, like in conventional optimization, the feasible region is defined via random set containments. All the 
above mentioned classes assure that the optimization problem has one objective function. If one considers several conflictual objective functions, one falls on the realm of fuzzy stochastic versions of multi objective programming problems $\left(C_{5}\right)$.

It is also worth mentioning that problems in classes $C_{1}, \cdots, C_{4}$ may be extended to the multi objective case, leading to $C_{1}^{l}, \cdots, C_{4}^{l}$ ( $I$ denoting the number of objectives considered). The reader is referred to [23] for an example of fuzzy stochastic multi objective model.

\subsection{Characteristics of FSO Models}

FSO models have the following common features:

1) Fuzziness and randomness are, in some way, involved in these models.

2) There is no agreed definition of optimum.

3) There are different perspectives about the problem situation.

4) The fuzzy stochastic optimization problem should be approximated by a deterministic one.

5) The closedness of the appropriate deterministic problem to the original one as well as the tractability of the former is desirable traits.

\section{Fuzzy Stochastic Optimization Methods}

\subsection{First Generation of FSO Methods}

Early attempts to solve decision problems involving fuzziness and randomness dated back to the 1980's [24] [25]. The notion of probabilistic set coupled to Bellman-Zadeh's confluence principle, served as the backdrop to these approaches. This line of research has been quickly extended to optimization problems with discrete random variables and vague relationships [26]. The above mentioned developments on FSO were followed by systematic comparisons between Stochastic Programming and Fuzzy Optimization [27]. These comparative studies displayed many similarities and differences that have been put in good use to deal with some complex stochastic programs through simple and relevant fuzzy optimization techniques [28]. By the same token, a method for incorporating random variables with flexible distributions in a mathematical program was obtained [29]. Research efforts have also been triggered for treating, in a synergetic way, fuzzy and stochastic constraints in a same Optimization problem [30]. Without a shadow of doubt, the concept of fuzzy random variable [31] has served as a catalyst that boosted FSO development. This hybrid tool provided a gold mine of opportunities for coping with situations where fuzziness and randomness co-occur in an optimization setting [32]. In the next section, we briefly survey solution procedures for Fuzzy Stochastic Optimization models mentioned in section 3 .

\subsection{Solution Procedures for FSO Models}

\subsubsection{General Procedure for Solving a FSO Model}

As suitable models are central to conceptual formulation of FSO problems, so 
suitable mathematical methods are central to their quantitative treatment. The presence of both possibilistic and probabilistic information within an Optimization framework is a harbinger of computational nightmares if one were to approach the problem without any simplifications. The game is to come up with approximations which tradeoff faithful representation of reality for computational tractability. Although the trajectory of FSO follows a situation-specific approach that do not lead to a generalization, we introduce below a general scheme from which most of existing FSO methods boil down. This algorithmic framework unifies a variety of seemingly different methods that have been derived from disparate approaches.

Before proceeding further, we need the following notations:

$D O$ : Transformation that converts the original problem into a form suitable for treatment.

If the problem at hand is ready for treatment, then $D O=I$ (identity transformation).

$D F$ : Deffuzification.

That is a process that associates to a given fuzzy system a deterministic counterpart.

$D R$ : Derandomization.

That is a procedure that immunizes from randomness.

$D F R$ : Transformation that deals simultaneously with fuzziness and randomness.

$D L(P)$ : stands for the problem resulting in applying $D L$ to $P$, where $L$ may be $O, F, R$ or $F R$.

We are now in a position to depict a general scheme for solving a FSO problem.

Step 0: make a choice of $D O, D F, D R, D F R$.

Step 1: Apply $T_{0}=D O$ to the original problem $(P)$ to obtain $T_{0}(P)$.

Step 2: Choose $T_{1} \in\{D F, D R, D F R\}$ and apply $T_{1}$ to $T_{0}(P)$ to obtain $T_{1}\left(T_{0}(P)\right)$.

Step 3: Choose $T_{2}$ as follows:

- If $T_{1}=D F R$ then $T_{2}=I$.

- Otherwise take $T_{2} \in\{D F, D R\}-T_{1}$.

- Apply $T_{2}$ to $T_{1}\left(T_{0}(P)\right)$ to get $T_{2}\left(T_{1}\left(T_{0}(P)\right)\right)$.

Step 4: Solve the resulting deterministic problem $T_{2}\left(T_{1}\left(T_{0}(P)\right)\right)$.

The choice in Step 0 can be fulfilled in many ways not all of them being equally satisfactory. This choice poses a double challenge. First, the transformations retained should not lead to a bad caricature of the reality. Secondly, they should be such that the resulting deterministic program could be computationally tractable. A FSO method becomes attractive if these two challenges are successfully overcome. Table 1 gives a sample of $D F$ and $D R$ transformations used in the literature for the case of linear constraints and are used respectively for fuzzy and random data. Moreover, $\tilde{c}_{\alpha}$ stands for the $\alpha$ cut of the fuzzy set $\tilde{c}$. 
Table 1. Deterministic counterparts of imprecise constraints.

\begin{tabular}{|c|c|c|}
\hline $\begin{array}{l}\text { Type of } \\
\text { constraints }\end{array}$ & Transformation & Deterministic counter part \\
\hline$\tilde{a} x \leq \tilde{b}$ & $D F_{1}$ & $D F_{1}(\tilde{a} x \leq \tilde{b})=\left\{x \mid \tilde{a}_{\alpha} x \leq \tilde{b}_{\alpha} ; \forall \alpha \in(0,1]\right\}$ \\
\hline$\tilde{a} x \leq \tilde{b}$ & $D F_{2}$ & $D F_{2}(\tilde{a} x \leq \tilde{b})=\left\{x \mid \tilde{a}_{\alpha_{i}} x \leq \tilde{b}_{\alpha_{i}} ; \alpha_{i} \in(0,1] ; i=1, \cdots, p\right\}$ \\
\hline \multirow[t]{4}{*}{$\tilde{a} x \leq \tilde{b}$} & $D F_{3}$ & $D F_{3}(\tilde{a} x \leq \tilde{b})=\{x \mid T(\tilde{a} x \leq \tilde{b}) \geq \beta\}$ \\
\hline & & where $T \in\{$ Poss, Nec $\}$ and $\beta$ is a prescribed \\
\hline & & threshold. \\
\hline & & $\begin{array}{l}\text { Poss and Nec stand for possibility and necessity } \\
\text { respectively. }\end{array}$ \\
\hline \multirow[t]{2}{*}{$\tilde{a} x \leq \tilde{b}$} & $D F_{4}$ & $D F_{4}(\tilde{a} x \leq \tilde{b})=\{x \mid \operatorname{Poss}(\tilde{a} x \leq \tilde{b}) \geq \alpha$ and $\operatorname{Nec}(\tilde{a} x \leq \tilde{b}) \geq \delta\}$ \\
\hline & & $\alpha$ and $\delta$ stand for prescribed thresholds. \\
\hline \multirow[t]{2}{*}{$\bar{a} x \leq \bar{b}$} & $D R_{1}$ & $D R_{1}(\bar{a} x \leq \bar{b})=\{x \mid(E \bar{a}) x \leq E(\bar{b})\}$ \\
\hline & & where $E$ stands for the expectation operator. \\
\hline \multirow[t]{2}{*}{$\bar{a} x \leq \bar{b}$} & $D R_{2}$ & $D R_{2}(\bar{a} x \leq \bar{b})=\{x \mid \operatorname{Prob}(\bar{a} x \leq \bar{b}) \geq \beta\}$ \\
\hline & & $\begin{array}{l}\text { where Prob is the probability measure and } \beta \text { a fixed } \\
\text { threshold. }\end{array}$ \\
\hline
\end{tabular}

For the sake of space, we briefly discuss in the section below, section (5.2.2). Only some approaches for models of different categories.

\subsubsection{Solving Approaches for Models in the Category $C_{1}$}

(a) Flexible programming with random data

Consider the following fuzzy stochastic model:

$$
\left(M_{1}\right)\left\{\begin{array}{l}
\min f(x, \tilde{r}) \\
g_{i}\left(x, \tilde{s}_{i}\right) \tilde{\leq} b_{i} ; i=1, \cdots, m \\
x \in X \subset \mathbb{R}^{n}
\end{array}\right.
$$

where $\tilde{r}, \tilde{s}_{i}$ are random variables on $(\Omega, F, P) ; f, g_{i}(i=1, \cdots, m)$ are functions of $\mathbb{R}^{n} \times \Omega$. "min" and " $\tilde{\leq}$ " indicate that the minimization and the inequality are not strict imperatives.

There are two main approaches for solving Problem $\left(M_{1}\right)$ in the literature. The symmetrical approach boils down from the general scheme depicted in section (5.2.1) by using the following transformations.

$T_{0}\left(M_{1}\right)=\left(M_{1}^{\prime}\right)$ where $\left(M_{1}^{\prime}\right)$ is the following problem. Find $x \in X$ such that:

$$
g_{i}\left(x, \bar{s}_{i}\right) \tilde{\leq} \bar{b}_{i} ; i=0,1, \cdots, m .
$$

Here $g_{0}=f, \bar{s}_{0}=\bar{r}$ and $\bar{b}_{0}$ plays the role of a fixed benchmark for the value of the objective function.

$$
T_{1}\left(M_{1}^{\prime}\right)=\left(M_{1}^{\prime \prime}\right) \text {, where }\left(M_{1}^{\prime \prime}\right) \text { is as follows. Find }
$$




$$
\arg \max _{x \in X}\left(\mu_{D}(x, \bar{s})\right)
$$

where

$$
\mu_{D}(x, \bar{s})=\wedge_{i=0}^{m} \mu_{i}\left(x, \bar{s}_{i}\right)
$$

and " $\wedge$ " stands for an operator used to translate the semantic meaning of the "and" connective.

It is worth noticing that $\mu_{i}(i=0,1, \cdots, m)$ are memberships functions of probabilistic sets representing the constraints:

$$
g_{i}\left(x, \overline{s_{i}}\right) \tilde{\leq} \bar{b}_{i} ; i=0,1, \cdots, m .
$$

$T_{1}\left(M_{1}^{\prime \prime}\right)=\left(M_{1}^{\prime \prime \prime}\right)$ where $\left(M_{1}^{\prime \prime \prime}\right)$ is obtained from $\left(M_{1}^{\prime \prime}\right)$ by immunizing from randomness through the expectation operator. That is $\left(M_{1}\right)^{\prime \prime \prime}$ is the following deterministic optimization problem.

$$
\max _{x \in X} E\left(\mu_{D}(x, \bar{s})\right) .
$$

Solving this optimization problem yields a satisfying solution of Problem $\left(M_{1}\right)$. The term symmetrical refers to the fact that the objective function and the constraints are considered as equivalent concepts.

To solve problem $\left(M_{1}\right)^{\prime \prime \prime}$, we need an analytical expression of the distribution of $\mu_{D}(x, \bar{s})$ denoted by $F_{\mu_{D}}(x, \bar{s})$. The following result, the proof of which may be found in [33], is helpful in this regard.

Theorem 1

(1) If $\mu_{D}(x, \omega)=\min _{i=0,1, \cdots, m} \mu_{i}(x, \omega)$ then

$$
\begin{aligned}
F_{\mu_{D}(x, \omega)}(z)= & \sum_{i=0}^{m} F_{\mu_{i}(x, \omega)}(z)-\sum_{j<k} F_{\mu_{j}(x, \omega) \mu_{k}(x, \omega)}(z, z) \\
& +\sum_{i<j<k} F_{\mu_{i}(x, \omega) \mu_{j}(x, \omega) \mu_{k}(x, \omega)}(z, z, z)+\cdots \\
& +(-1)^{m-2} F_{\mu_{0}(x, \omega) \mu_{1}(x, \omega) \cdots \mu_{m}(x, \omega)}(z, \cdots, z) .
\end{aligned}
$$

(2) If $\mu_{D}(x, \omega)=\gamma \min _{i} \mu_{i}(x, \omega)+(1-\gamma) \min \left(1, \sum_{i} \mu_{i}(x, \omega)\right)$, where $\gamma$ stands for a coefficient of compensation ranging between 0 and 1 , then

$$
F_{\mu_{D}(x, \omega)}(z)=L^{-1}\left(g_{1}(s) \cdot g_{2}(s)\right),
$$

where

$$
\begin{gathered}
g_{1}(s)=\int_{0}^{\infty} \mathrm{e}^{-\left(\frac{s}{\gamma}\right) z} F_{\min _{i} \mu_{i}(x, \omega)}(z) \mathrm{d} z, \\
g_{2}(s)=\int_{0}^{\infty} \mathrm{e}^{-\left(\frac{s}{1-\gamma}\right) z} F_{\min \left(1, \Sigma_{i} \mu_{i}(x, \omega)\right)}(z) \mathrm{d} z
\end{gathered}
$$

and $L^{-1}$ stands for the inverse of the Laplace transform.

For a perspective on the asymmetrical approaches for solving Problem $\left(M_{1}\right)$. We refer the reader to [34]. Asymmetrical approaches have the advantage of confining the objective function to its classical role of ranking alternatives and the constraints to their role of delineating the feasible set. Ongoing research includes the use of other more effective paradigms, like the chance constrained or 
the multistage ones instead of merely sticking on the expectation operator to immunize from randomness. Another issue researchers are busy considering is the trade-offs between the two classes of approaches. Results along this line would make it possible to decide which approach is most suitable under given circumstances.

\section{(b) Mathematical programming with fuzzy random coefficients}

Advances on elucidating properties of fuzzy random variables have triggered considerable work on how to deal with mathematical programs with fuzzy random (fr) or random fuzzy (rf) coefficients. It is worth mentioning that the general scheme described in section (5.2.1) applies here as well. Typical, but by no means unique, transformations are used. To provide a taste of what is done on optimization with fuzzy random data, we consider the following Program $\left(M_{2}\right)$. We deliberately restrict ourselves to the linear case so that basic ideas are illustrated in a relatively simple context. For non linear programs, we refer the reader to [34] [35].

Consider the following fuzzy stochastic program:

$$
\left(M_{2}\right)\left\{\begin{array}{l}
\max c x \\
\sum \tilde{a}_{i j} x_{j} \leq \tilde{b}_{i} ; i=1, \cdots, m \\
x_{j} \geq 0
\end{array}\right.
$$

Let

$$
H_{\alpha}(\omega)=\left(\tilde{A}_{\alpha}(\omega), \tilde{b}_{\alpha}(\omega)\right), H_{\alpha}^{+}(\omega)=\left(\bar{A}_{\alpha}(\omega), \bar{b}_{\alpha}(\omega)\right),
$$

and

$$
H_{\alpha}^{-}(\omega)=\left(\underline{A}_{\alpha}(\omega), \underline{b}_{\alpha}(\omega)\right),
$$

where

$$
\tilde{A}_{\alpha}(\omega), \bar{A}_{\alpha}(\omega), \underline{A}_{\alpha}(\omega)
$$

stand for matrices

$$
\left(\tilde{a}_{i j \omega \alpha}\right)_{i, j},\left(\bar{a}_{i j \omega \alpha}\right)_{i, j},\left(\underline{a}_{i j \omega \alpha}\right)_{i, j}
$$

respectively and $\tilde{a}_{i j \omega \alpha}$ denotes the $\alpha$-level set of $\tilde{a}_{i j \omega}$, while $\bar{a}_{i j \omega \alpha}, \underline{a}_{i j \omega \alpha}$ stand for the upper and lower endpoints of $\tilde{a}_{i j \omega \alpha}$ respectively. Similarly, $\tilde{b}_{\alpha}(\omega), \bar{b}_{\alpha}(\omega)$ and $\underline{b}_{\alpha}(\omega)$ stands for vectors:

$$
\left(\begin{array}{c}
\tilde{b}_{1 \omega \alpha} \\
\vdots \\
\tilde{b}_{m \omega \alpha}
\end{array}\right),\left(\begin{array}{c}
\bar{b}_{1 \omega \alpha} \\
\vdots \\
\bar{b}_{m \omega \alpha}
\end{array}\right),\left(\begin{array}{c}
\underline{b}_{1 \omega \alpha} \\
\vdots \\
\underline{b}_{m \omega \alpha}
\end{array}\right)
$$

respectively. Moreover $W$ denotes the optimum of Problem $\left(M_{2}\right)$ and $W_{\alpha}^{*}$ stands for the optimum value of $\left(M_{2}^{\alpha *}\right)$, where $* \in\{-,+\}$ and $\left(M_{2}^{\alpha-}\right)$ and $\left(M_{2}^{\alpha+}\right)$ are the following programs respectively:

$$
\left(M_{2}^{\alpha-}\right)\left\{\begin{array}{l}
\max c x \\
\underline{A}_{\alpha}(\omega) x \leq \underline{b}_{\alpha}(\omega) \\
x \geq 0
\end{array}\right.
$$




$$
\left(M_{2}^{\alpha+}\right)\left\{\begin{array}{l}
\max c x \\
\tilde{A}_{\alpha}(\omega) x \leq \tilde{b}_{\alpha}(\omega) \\
x \geq 0
\end{array}\right.
$$

$g_{i}^{\alpha r}$ is the penalty for unit of discrepancy between $\sum \bar{a}_{i j \omega \alpha r}$ and $\underline{b}_{i \omega \alpha r}$. $\beta$ stands for a prescribed threshold fixed by the Decision Maker. $f_{H_{\alpha}^{*}}$ denotes the density function of $H_{\alpha}^{*}(* \in\{-,+\})$ and $\left(V_{\alpha}^{*}\right)_{i}$ is the $i^{\text {th }}$ decision region of $\left(M_{2}^{\alpha *}\right)(* \in\{-,+\})$.

All transformations used in this table are well explained in Table 2 except DR3, DR4 and DR5 that are respectively the two stages, the random simplex and the distribution paradigms applied to a stochastic program. For solving resulting deterministic problems, one use techniques from simple ones like the simple method [40] to sophisticated ones like hybrid intelligent algorithms [41] via metaheuristics, like Genetic algorithms [42] and Tabu search [43]. A quick look at Table 2 may convey the wrong impression that methods listed on that table do not offer much scope for theoretical results. Upon closer examination one may realize that these approaches are not pedestrian or brute force methods. They rely on deep theoretical insights. We mention below some of them.

It is well known that constraints on probability induce non convexities which preclude application of powerful convex optimization techniques. So the viability of approach 1 in Table 2 depends on whether it is possible to convert constraints

Table 2. Some instances of approaches for solving (2).

\begin{tabular}{|c|c|c|c|c|}
\hline \multicolumn{2}{|c|}{ Transformation } & \multirow{2}{*}{ - Resulting deterministic problem $\left(P^{\prime}\right)$} & \multirow{2}{*}{ Approach } & \multirow{2}{*}{ references } \\
\hline$T_{1}$ & $T_{2}$ & & & \\
\hline$D F_{2}$ & $D R_{2}$ & $\begin{array}{l}\max c x \\
\operatorname{Prob}\left[\sum_{j=1}^{n} \bar{a}_{i j \omega \alpha r} x_{j} \leq \underline{b}_{i \omega \alpha r}\right] \geq \beta, \\
r=1, \cdots, p ; i=1, \cdots, m ; x_{j} \geq 0 \\
j=1, \cdots, n\end{array}$ & 1 & {$[36]$} \\
\hline$D F_{4}$ & $D R_{5}$ & $\begin{array}{l}\text { Find the fuzzy probability } \\
\text { distribution of } W \text { and its } \\
\text { expectation given respectively by: } \\
\tilde{F}_{W}(x)=\bigcup_{\alpha \in(0,1]} \alpha\left[F_{H_{\alpha^{+}}}(x), F_{H_{\alpha^{-}}}(x)\right] \\
\tilde{E}_{W}(x)=\bigcup_{\alpha \in(0,1]} \alpha\left[E\left(H_{\alpha}^{-}\right), E\left(H_{\alpha}^{+}\right)\right]\end{array}$ & 2 & {$[37]$} \\
\hline$D F_{2}$ & $D R_{3}$ & $\begin{array}{l}\max \left[c X-E\left(\sum_{r=1}^{p} \sum_{i=1}^{m} g_{i}^{\alpha r} y_{i}^{\alpha r}(\omega)\right)\right] \\
y_{i}^{\alpha r}(\omega)=\sum_{j=1}^{n} \bar{a}_{i j \omega \alpha r} x_{j}+\underline{b}_{i \omega \alpha r}\end{array}$ & 3 & {$[38]$} \\
\hline$D F_{4}$ & $D R_{4}$ & $\begin{array}{l}\text { Use the random simplex method to } \\
\text { find } x_{\alpha} \text { solution of max } c x \\
\underline{a} \alpha(\omega) x \leq \underline{b} \alpha(\omega) ; \bar{a}_{\alpha}(\omega) x \leq \bar{b}_{\alpha} \\
\alpha \in(0,1] \text { and construct } x^{*}=\bigcup_{\alpha \in(0,1]} \alpha x_{\alpha}\end{array}$ & 4 & {$[39]$} \\
\hline
\end{tabular}


on probability on a feasible convex set. Moreover, approach 2 relies on the following result.

Theorem 2. Consider the Problem $(P)$ and all related notation below.

$$
(P) \begin{cases}\max c x, & \\ \sum_{j=1}^{n} a_{i j}^{*} x_{j} \leq b_{i}^{*}, & i=1, \cdots, m, \\ x_{j} \geq 0, & j=1, \cdots, n,\end{cases}
$$

where $c, x \in \mathbb{R}^{n}$ and * means that $a_{i j}$ and $b_{i}$ are either fuzzy quantities or random variables, “*” takes "+" if the data is random and it takes "-" if the data is fuzzy; then the distribution of $H_{\alpha}^{*}$ and its expectation are respectively:

$$
\begin{gathered}
F_{H_{\alpha}^{*}}(x)=\sum_{i=1}^{R} \int_{\left\{H^{*}(\omega) \mid H_{\alpha}^{*}(\omega) \in\left(V_{\alpha}^{*}\right)_{i} \text { and } W_{\alpha}^{*}<x\right\}} f_{H_{\alpha}^{*}}(s) \mathrm{d} s \\
E\left(H_{\alpha}^{*}\right)=\sum_{i=1}^{R} \int_{\left(V_{\alpha}^{*}\right)_{i}} W_{\alpha}^{*} f_{H_{\alpha}^{*}}(s) \mathrm{d} s .
\end{gathered}
$$

For the proof of this result, we invite the reader to consult [33]. We must also stress the fact that several methods have been developed over the past few decades due to the efforts of many researches, e.g. [44] [45]. Surveying all these developments is however beyond the scope of this paper.

Important part of research in this realm includes the generalization of existing ideas by using other transformations and the driving up to the performance analysis in the side of effectiveness rather than sticking on computational efficiency. Something that should also be pursued with focused attention is the search for solutions of fuzzy stochastic nature, in a way to reflect the fuzzy random structure of the problem.

The case where one has both fuzzy and random data in a same optimization problem, can be converted into the case one has fuzzy random data, by considering involved fuzzy and random quantities as degenerate fuzzy random variables (e.g. [46]). Another notion that has energized FSO development is that of random fuzzy variable [47]. This notion that has been moulded by Liu [48], generalizes quite naturally the notion of random variable. A fascinating approach based on this notion and fuzzy random simulation that integrates neural network and genetic algorithm has been pushed forward in [49], to solve a mathematical program with random fuzzy parameters.

In addition to shedding light on analysis of Optimization problems under hybrid uncertainty, the proposed algorithm may be the only one resort for solving optimization problems under randomness and fuzziness, where random fuzzy variable is the only one format available for data. It is our belief that exploring the duality between a mathematical program with fuzzy random data and its counterpart with related random fuzzy parameters, will help fostering a more deep understanding of characteristics of Fuzzy Stochastic Optimization problems.

(c) Mathematical program with random variables having fuzzy parameters

We now move to solution procedures for optimization problems, where another specific tool, namely uncertain probability, is used for modeling hybrid 
imprecision in the data of the problem. The mathematical program on which ideas will be illustrated is as follows.

$$
\left(M_{3}\right)\left\{\begin{array}{l}
\min c x \\
A_{i}^{*} x \leq b_{i}^{*} ; i=1, \cdots, m \\
x \in X=\left\{x \in \mathbb{R}^{n} \mid x \geq 0\right\}
\end{array}\right.
$$

where " $*$ " means the daturn is random with some fuzzy parameters. This model has many applications in situations where experts who provide data of the optimization problem feel more comfortable in coupling their vague perception with hard statistical data. To this end, they may prefer to represent these data in the form of random variables with vague parameters. As an example, consider a portfolio selection problem where, due to stock expects' judgments and investors' different opinions, the security returns are modeled as random variables with fuzzy parameters. Model $\left(M_{3}\right)$ has not received much attention in the literature. The art here is to find an approximation scheme simple enough to be computable, but not so simple that useful detail is lost. This is done in [50] by making use of the fuzzy version of the well known chance constrained programming approach. Uncertain probabilities of constraints are enforced to be larger than a specified level fixed by the Decision maker.

This means one has to consider the following problem.

$$
\left(M_{3}^{\prime}\right)\left\{\begin{array}{l}
\min c x \\
\tilde{P}\left[\sum_{j=1}^{n} a_{i j}^{*} x \leq b_{i}^{*}\right] \geq \tilde{\delta}_{i} ; i=1, \cdots, m \\
x \in X
\end{array}\right.
$$

where $\tilde{P}$ stands for uncertain probability and $\tilde{\delta}_{i}, i=1, \cdots, m$ are fuzzy thresholds fixed by the Decision maker. The question that immediately arises is that of converting Problem $\left(M_{3}^{\prime}\right)$ into a standard mathematical program.

Fascinating results in connection with this issue have been obtained in the literature. For the sake of space, we only give below one of these results. To this end, we need the following lemma.

Lemma 1. Assume $a_{i j}^{*}(j=1, \cdots, n)$ and $b_{i}^{*} ; i=1, \cdots, m$ are independent and normally distributed random variables with fuzzy parameters. Put

$$
\xi_{i}^{*}(x)=\sum_{j=1}^{n}\left(a_{i j}^{*} x_{j}-b_{i}^{*}\right)
$$

Then $\xi_{i}^{*}(x)$ is also normally distributed with fuzzy mean $\tilde{m}_{\xi_{i}}(x)$ and fuzzy variance $\tilde{\sigma}_{\xi_{i}}^{2}(x)$.

Let now the fuzzy numbers $\tilde{\delta}_{i} ; i=1, \cdots, m$ be threshold fixed by the Decision maker. As $\tilde{m}_{\xi_{i}}(x), \tilde{\sigma}_{\bar{s}_{i}}^{2}(x)$ and $\tilde{\delta}_{i}$ are fuzzy numbers, their $\alpha$-cuts are intervals denoted respectively as follows.

$$
\begin{aligned}
\tilde{m}_{\xi_{i}}^{\alpha}(x) & =\left[m_{\xi_{i}}^{\alpha L}(x), m_{\xi_{i}}^{\alpha U}(x)\right] \\
\tilde{\sigma}_{\xi_{i}}^{2}(x) & =\left[\sigma_{\xi_{i}}^{2 \alpha L}(x), \sigma_{\xi_{i}}^{2 \alpha U}(x)\right] \\
\tilde{\delta} & =\left[\delta_{i}^{\alpha L}, \delta_{i}^{\alpha U}\right] .
\end{aligned}
$$


Theorem 3. Under assumption of Lemma (1), Problem $\left(M_{3}^{\prime}\right)$ is equivalent to the following mathematical program:

$$
\left(M_{3}^{\prime \prime}\right)\left\{\begin{array}{l}
\min c x \\
\phi\left(\frac{-m_{\xi_{i}}^{\alpha U}(x)}{\sigma_{\xi_{i}}^{2 \alpha U}(x)}\right) \geq \delta_{i}^{\alpha U} ; i=1, \cdots, m \\
x \in X ; \alpha \in(0,1]
\end{array}\right.
$$

where $\phi$ stands for the density function of the standard normal distribution.

It is worth noting that Problem $\left(M_{3}^{\prime \prime}\right)$ has infinitely many constraints and it can be handled by semi-infinite mathematical programming techniques [49]. The prevailing research directions in this setting include analysis of non linear variants of Problem $\left(M_{3}\right)$ along with methods for solving them and consideration of other distribution than the normal one. Another worthy avenue for research in this framework, is to carry out case studies in a way to validate existing approaches.

\subsubsection{A method for Solving a Model in the Category $C_{4}$}

One key ingredient for dealing with models in the category $C_{4}$ is the lattice isomorphism between the set of fuzzy random sets and the family of their $\alpha$-level sets [51]. It makes a lot of sense to take a defuzification approach, the transformation that immunizes the original robust fuzzy stochastic program from fuzziness by making use of the above mentioned isomorphism and by exploring the fact that $\alpha$-level sets of a fuzzy random set are random intervals. A suitable derandomization procedure would then be a stochastic programming technique like $D R_{1}, D R_{2}$ or $D R_{3}$ (see Table 1 ).

Here is an example of a fuzzy stochastic robust programming model.

$$
\left(P_{R}\right)\left\{\begin{array}{l}
\max c x \\
\underline{A}_{1} x_{1}+\underline{A}_{2} x_{2}+\cdots+\underline{A}_{n} x_{n} \subseteq \underline{B} \\
x_{1} \geq 0, x_{2} \geq 0, \cdots, x_{n} \geq 0
\end{array}\right.
$$

where $c, x \in \mathbb{R}^{n}$ and $\underline{A}, \underline{B}$ are fuzzy sets of $\mathbb{R}^{n}$. Components of $\underline{A}$ denoted by $\underline{a}_{i j}$ are fuzzy numbers, while components of $B$ denoted by $\underline{b}_{i}$ are convex fuzzy sets of $\mathbb{R}$ having random characteristics. More precisely, we assume like in [51] that the endpoints of an $\alpha$-level of $b_{i}$ are random variables with known probability distributions. It is well known that Problem $\left(P_{R}\right)$ is equivalent to the following optimization problem:

$$
\left(P_{R}^{\prime}\right)\left\{\begin{array}{l}
\max c x \\
\underline{A}_{1 \alpha} x_{1}+\cdots+\underline{A}_{n \alpha} x_{n} \subseteq B_{\alpha}, \forall \alpha \in(0,1] \\
x_{1} \geq 0, x_{2} \geq 0, \cdots, x_{n} \geq 0
\end{array}\right.
$$

To tackle randomness surrounding $\underline{B}$, we resort to chance constrained programming approach. This approach requires that the constraints need to be satisfied with some higher probability. For instance, for a stochastic constraint:

$$
D(\omega) x \leq e(\omega)
$$


One may require that

$$
\operatorname{Pr}[\{\omega \mid D(\omega) x \leq e(\omega)\}] \leq 1-l
$$

where $l$ is a fixed level of probability. Constraint (2) can be merely written:

$$
D(\omega) x \leq e(\omega)^{(l)}
$$

where $e(\omega)^{(l)}=F^{-L}(l) \cdot F$ being the cumulative distribution of $e$. For details on Chance Constrained programming, we refer the reader to [52]. It is also well known that $\alpha$-levels of $\underline{a}_{i j}$ and $\underline{b}_{i}$ are real intervals. We denote them by $\left[\underline{a}_{i j \alpha}, \bar{a}_{i j \alpha}\right]$ and $\left[\underline{b}_{i \alpha}, \bar{b}_{i \alpha}\right]$ respectively. Making use of the operations on the set of real bounded intervals and resorting at the chance constrained programming technique to deal with randomness, the Problem $\left(P_{R}^{\prime}\right)$ becomes:

$$
\left(P_{R}^{\prime \prime}\right)\left\{\begin{array}{l}
\max c x \\
\sum_{j=1}^{n} \underline{a}_{i j \alpha} x_{j} \geq \underline{b}_{i \alpha}^{(l)} ; i=1, \cdots, m ; \alpha \in(0,1] \\
\sum_{j=1}^{n} \bar{a}_{i j \alpha} x_{j} \leq \bar{b}_{i \alpha}^{(l)} ; i=1, \cdots, m ; \alpha \in(0,1] \\
x_{j} \geq 0 ; j=1, \cdots, n
\end{array}\right.
$$

This resulting program is a semi-infinite optimization problem and complex optimization techniques are required to solve it. Nevertheless, if the image of the membership function of $\underline{b}_{i}$ denoted by $\left(\operatorname{Im}_{\mu_{\bar{b}_{i}}}\right)$ is finite for every $i$, say $\operatorname{Im}_{\mu_{\overline{b_{i}}}}=\left\{\alpha_{1 i}, \cdots, \alpha_{k i}\right\}$ then (see [53]), Problem $\left(P_{R}^{\prime \prime}\right)$ becomes merely:

$$
\left(P_{R}^{\prime \prime \prime}\right)\left\{\begin{array}{l}
\max c x \\
\sum_{j=1}^{n} \underline{a}_{i j \alpha_{s_{i}}} x_{j} \geq \underline{b}_{i \alpha_{s_{i}}}^{(l)} ; i=1, \cdots, m ; s_{i}=1, \cdots, k_{i} \\
\sum_{j=1}^{n} \bar{a}_{i j \alpha_{s_{i}}} x_{j} \leq \bar{b}_{i \alpha_{s_{i}}}^{(l)} ; i=1, \cdots, m ; s_{i}=1, \cdots, k_{i} \\
x_{j} \geq 0 ; j=1, \cdots, n
\end{array}\right.
$$

Problem $\left(P_{R}^{\prime \prime \prime}\right)$ is a standard linear program, that can be solved by existing linear programming software.

\subsubsection{A method for Solving a Model in the Category $C_{2}$}

To illustrate how a Fuzzy Stochastic dynamic model can be solved, we briefly discuss a multistage decision process, involving randomness and fuzziness. The state space of the process at hand is

$$
X=\left\{x_{1}, \cdots, x_{k}, x_{k+1}, \cdots, x_{n}\right\} .
$$

We assume that the termination set, denoted by $T$, consists of states $x_{k+1}, \cdots, x_{n}$ and we put $\bar{T}=X / T$. We also assume that the constraints of the process are defined through state-dependent fuzzy sets $C\left(x_{k}\right)$ over the control space $U$. The membership function of $C\left(x_{k}\right)$ is denoted by $\mu_{C}\left(\mu_{t} \mid x_{t}\right)$. The conditional probabilities of a state transition under a control $\mu_{t}$ are given as follows.

$$
p\left(x_{t+1} \mid x_{t}, \mu_{t}\right)= \begin{cases}1 & \text { if } x_{t+1}=x_{t} \in T \\ 0 & \text { if } x_{t+1}=x_{t} \in T \\ 0 & \text { if } x_{t} \in T, x_{t+1} \neq x_{t}\end{cases}
$$


Suppose now that the sequent inputs $u_{0}, u_{1}, \cdots, \mu_{t}, \cdots$ are determined by a stationary policy function $\Pi: \bar{T} \rightarrow U$. For a policy $\Pi$, in a given state $x_{i} \in \bar{T}$, the decision $D_{\Pi}(x t)$ with respect to $\Pi$ is the confluence of the constraint in the transition from $x_{t}$ to $x_{t+1}, C_{\Pi}\left(x_{i}\right)$ and the next decision $D_{\Pi}\left(x_{t+1}\right)$ with respect to $\Pi$. If $x_{t+1} \in T$, we set $D_{\Pi}\left(x_{t+1}\right)=G\left(x_{t+1}\right)$ for any $\Pi$.

Given $x_{t}$ the state $x_{t+1}$ is a random variable characterized by the conditional probability function $p\left(x_{t+1} \mid x_{t}, u_{t}\right)$. Thus

$$
D_{\Pi}\left(x_{t}\right)=C_{\Pi}\left(x_{t}\right) \cap E D_{\Pi}\left(x_{t+1}\right)
$$

or

$$
u_{D}\left(x_{t} \mid \Pi\right)=u_{C}\left(\Pi\left(x_{t}\right) \mid x_{t}\right) \cap E u_{D}\left(x_{t+1} \mid \Pi\right)
$$

where

$$
\begin{aligned}
E u_{D}\left(x_{t+1} \mid \Pi\right)= & \sum_{x_{t+1} \in \bar{T}} p\left(x_{t+1} \mid x_{t}, \Pi\left(x_{t}\right)\right) \cdot u_{D}\left(x_{t+1} \mid \Pi\right) \\
& +\sum_{x_{t+1} \in T} p\left(x_{t+1} \mid x_{t}, \Pi\left(x_{t}\right)\right) \cdot u_{G}\left(x_{t+1}\right)
\end{aligned}
$$

and hence,

$$
\begin{aligned}
u_{D}\left(x_{t} \mid \Pi\right)= & u_{C}\left(\Pi\left(x_{t}\right)\right) \wedge\left[\sum_{x_{t+1} \in \bar{T}} p\left(x_{t+1} \mid x_{t}, \Pi\left(x_{t}\right)\right) \cdot u_{D}\left(x_{t+1} \mid \Pi\right)\right. \\
& \left.+\sum_{x_{t+1} \in T} p\left(x_{t+1} \mid x_{t}, \Pi\left(x_{t}\right)\right) \cdot u_{G}\left(x_{t+1}\right)\right] .
\end{aligned}
$$

Given a policy $\Pi=\left(\Pi\left(x_{1}\right), \cdots, \Pi\left(x_{k}\right)\right)$ we consider the following vectors:

$$
\begin{gathered}
u_{C}(\Pi)=\left(u_{C}\left(\Pi\left(x_{1}\right) \mid x_{1}\right), \cdots, u_{C}\left(\Pi\left(x_{k}\right)\right) x_{k}\right)(\text { constraint vector) } \\
u_{D}(\Pi)=\left(u_{D}\left(x_{1} \mid \Pi\right), \cdots, u_{D}\left(x_{k} \mid \Pi\right)\right)(\text { control vector) } \\
u_{G}=\left(u_{G}\left(x_{k+1}\right), \cdots, u_{G}\left(x_{n}\right)\right) \text { (goal vector) }
\end{gathered}
$$

Let' also introduced the following transition matrices:

$$
\begin{gathered}
P_{\bar{T}}(\Pi)=\left(p_{i j}(\Pi)\right)_{i}, j=1, \cdots, k \\
P_{T}(\Pi)=\left(p_{i j}(\Pi)\right)_{j=k+1, \cdots, n}^{i=1, \cdots, k}
\end{gathered}
$$

with these notations, the system of equations in (3) can be put in a more compact form.

$$
u_{D}(\Pi)=u_{C}(\Pi) \wedge\left[P_{\bar{T}}(\Pi) \cdot u_{D}(\Pi)+P_{T}(u) \cdot u_{G}\right]
$$

$\Pi$ is said to be proper, if there is a natural number $K$ such that $\left\|p_{\bar{T}}^{K}\right\|<1,1 \leq K \leq k$ where $k$ is the number of states in $\bar{T}$. The set of proper policies is denoted by $\Pi_{p} . \Pi_{p}$ is a finite set. So we may write $\Pi_{p}=\left\{\Pi^{1}, \cdots, \Pi^{r}\right\}$, where $r$ is finite. An optimal policy denoted by $\Pi^{\text {opt }}$ is the one that maximizes $u_{D}(\Pi)$. It is shown that under mild assumptions, the termination set $T$ is reachable from any initial state in $\bar{T}$. Moreover, an optimal policy $\Pi^{\text {opt }} \in \Pi_{p}$ can be obtained from the solution of the following fixed point problem: 


$$
V(\omega)=\vee_{v=1}^{r}\left\{C_{v} \wedge\left[A_{v} \omega+b_{v}\right]\right\}
$$

where $\omega, A_{v}, b_{v}$ and $c_{v}$ stand for $u_{D}(\Pi), P_{\bar{T}}\left(\Pi^{v}\right), P_{T}\left(\Pi^{v}\right) \cdot u_{G}$ and $u_{C}\left(\Pi^{v}\right)$ respectively.

The literature on multiperiod programming under fuzziness and randomness is rather limited. This could be due to the lack of tractable methodologies. The optimality principle, so profusely used in deterministic dynamic programming, does not lend itself better in a fuzzy stochastic environment. Nevertheless attempt to integrate fuzziness and randomness in a dynamic programming framework is found in [54]. In that paper, authors described an approach for solving a dynamic programming involving stochastic state transition, fuzzy constraints, fuzzy goal and an implicitely defined termination time. This approach relies heavily on the Bellman-Zadeh's confluence principle [37] [55]. Some interesting results on the robustness of solutions obtained by this way are also outlined. Another approach which deserves more emphasis has been presented by Ganji et al. [56]. Here the picture is made even more complicated by the presence of different decision-making mind-sets. More recently, a new approach named stochastic fuzzy neural network [57] has been introduced. It aims at overcoming some of the limitations of the traditional methods like the difficulties related to the curse of dimensionality due to the increase in the number of decision and state variables. This approach consists of training stochastically a neuro fuzzy system, in a way to represent the system operational strategy.

The prevailing research direction in the Fuzzy Stochastic dynamic programming framework is the improvement of this Neural network approach in a way to address drawbacks of existing models like the impossibility of considering simultaneously related fuzzy and stochastic characteristics.

\subsubsection{Algorithms for Dealing with Models in Category $C_{3}$}

Hierarchical decision making process is extremely practical to such decentralized systems as agriculture, government policy, economic systems, finance, etc. For more realism on their multilevel decision models, researches have investigated ways for incorporating fuzziness into these settings. Related solution concepts like Stackelberg strategy have been elaborated and interesting results have been obtained. Powerful algorithms for singling out desired solutions have also been pushed forward. These approaches are at the root of extensions to Fuzzy stochastic multilevel methodologies. In a method that integrate fuzzy random simulation, neural network training and Genetic algorithm has been developed to find a satisfying solution of a fuzzy random multilevel program. Another interesting approach for the two-level case is found. Here essential ingredients used are fuzzy random versions of the Nash and the Stackelberg-Nash equilibria. The former is used for the lower level problem, while the latter is used for the overall problem. These approaches give rise to a number of challenging issues including effectiveness and convergence of proposed methods. Currently researchers are busy pondering these thorny issues. 
In a way to illustrate how a multilevel optimization problem under randomness and fuzziness can be solved, we briefly discuss below an approach proposed by Sakawa, Nishizaki and Katagiri [58] for a two-level linear programming problem.

To illustrate how algorithms for dealing with Fuzzy stochastic multilevel programs work, consider the following optimization problem:

$$
\left(P_{M L}\right)\left\{\begin{array}{l}
\operatorname{Minimize}\left(\tilde{\bar{C}}_{11} x_{1}+\tilde{\bar{C}}_{12} x_{2}\right) \text { for } D M_{1} \\
\operatorname{Minimize}\left(\tilde{\bar{C}}_{21} x_{1}+\tilde{\bar{C}}_{22} x_{2}\right) \text { for } D M_{2} \\
\text { subject to : } A x \leq b ; x \geq 0,
\end{array}\right.
$$

where $D M_{i}$ stands for Decision maker $i, \tilde{\bar{C}}_{l j}, l=1,2 ; j=1,2$ are fuzzy random variables defined on a probability space $(\Omega, \mathcal{F}, P)$ and whose realized values $\tilde{\bar{C}}_{l j}(\omega), l=1,2 ; j=1,2$ are fuzzy numbers with membership functions of the form:

$$
u_{\tilde{\bar{C}}_{i j}(\omega)}(t)= \begin{cases}L\left(\frac{d_{l j}(\omega)-t}{\beta_{l j}}\right) & \text { if } t \leq d_{l j}(\omega) \\ R\left(\frac{t-d_{l j}(\omega)}{\delta_{l j}}\right) & \text { if } t>d_{l j}(\omega)\end{cases}
$$

where $L$ and $R$ are respectively non decreasing and non increasing functions of $\mathbb{R}$ such that $L(0)=R(0)=1$ and $d_{l j}(\omega), \beta_{l j}(\omega)$ and $\delta_{l j}(\omega)$ are respectively the center value, the left spread value and the right spread value of $\tilde{\bar{C}}_{l j}(\omega)$. Using Zadeh extension principle; we have that:

$$
u_{\tilde{\bar{C}}_{l j}(\omega) x}(v)= \begin{cases}L\left(\frac{d_{l}(\omega) x-v}{\beta_{l} x}\right) & \text { if } v \leq d_{l}(\omega) x \\ R\left(\frac{v-d_{l}(\omega) x}{\delta_{l} x}\right) & \text { if } v>d_{l}(\omega) x\end{cases}
$$

where $d_{l}(\omega)=\left(d_{l 1}(\omega), d_{l 2}(\omega)\right), \quad \beta_{l}=\left(\beta_{l 1}, \beta_{l 2}\right) \quad$ and $\quad \delta_{l}=\left(\delta_{l 1}, \delta_{l 2}\right) ; l=1,2$. Suppose that the decision makers are happy in minimizing their objective functions under the condition that all coefficients $\tilde{\bar{C}}_{l j}(\omega), l=1,2 ; j=1,2$ belongs to $\alpha$-level sets of corresponding fuzzy random variables, for a fixed $\alpha$. Then Problem $\left(P_{M L}\right)$ reads:

$$
\left(P_{M L}^{\prime}\right)\left\{\begin{array}{l}
\text { Minimize } \bar{C}_{1} x \text { for } D M_{1} \\
\text { Minimize } \bar{C}_{2} x \text { for } D M_{2} \\
\text { subject to }: x \in X=\left\{x \in \mathbb{R}^{2} \mid A x \leq b ; x \geq 0\right\} \\
\bar{C}_{1 j} \in \tilde{\bar{C}}_{1 j \alpha}, \tilde{\bar{C}}_{2 j} \in \tilde{\bar{C}}_{2 j \alpha} ; j=1,2 .
\end{array}\right.
$$

To handle imprecision inherent to objective functions, we consider fuzzy goals, say the objective value should be substantially less than some given value. These goals are elicited by $D M_{1}$ and $D M_{2}$. Assume the membership functions of these goals are $\mu_{1}$ and $\mu_{2}$ for objective 1 and objective 2 respectively. It is 
worth mentioning that $\mu_{l}\left(\tilde{\bar{C}}_{l}(\omega) x\right), l=1,2$ vary randomly. A deterministic counterpart of Problem $\left(P_{M L}^{\prime}\right)$ may be obtained by adopting the following strategy, closely related to chance constrained programming. The strategy is to require that the probability that the degrees of satisfaction of fuzzy goals $\mu_{l}\left(\tilde{\bar{C}}_{l}(\omega) x\right), l=1,2$ be greater to some optimal values $\left(\max h_{1}, \max h_{2}\right)$ be not less than some fixed permissible levels $\left(\theta_{1}, \theta_{2}\right)$. With these requirements, Problem $\left(P_{M L}^{\prime}\right)$ can be written:

$$
\left(P_{M L}^{\prime \prime}\right)\left\{\begin{array}{l}
\text { Maximize } h_{1} \text { for } D M_{1} \\
\text { Maximize } h_{2} \text { for } D M_{2} \\
\text { subject to }: P\left(\left\{\omega \mid \mu_{1}\left(\tilde{\bar{C}}_{1}(\omega) x\right) \geq h_{1}\right\}\right) \geq \theta_{1} \\
P\left(\left\{\omega \mid \mu_{2}\left(\tilde{\bar{C}}_{2}(\omega) x\right) \geq h_{2}\right\}\right) \geq \theta_{2} \\
x \in X ; C_{l j} \in \tilde{\bar{C}}_{l j \alpha}^{l} ; l=1,2 ; j=1,2 .
\end{array}\right.
$$

where $h_{l}, l=1,2$ are target values and $\theta_{l}, l=1,2$ are permissible probability levels, specified by the decision makers. Before discussing how to find a satisfying solution of the original multilevel program using the auxiliary problem $\left(P_{M L}^{\prime \prime}\right)$, we need the following notations. We assume that the random parameter associated to the fuzzy random variable $\tilde{\bar{C}}_{l}$ is denoted by $\bar{d}_{l}$ and is expressed as $\bar{d}_{l}=d_{l}^{1}+\bar{t}_{l} d_{l}^{2}$, where $\bar{t}_{l}$ is a random variable with probability distribution $T_{l}$. Moreover, we denote by $F^{*}$ the pseudo inverse of a real-valued function $F$ of $\mathbb{R}$. That is for $\theta \in \mathbb{R}, F^{*}(\theta)=\inf \{r \in \mathbb{R} \mid F(r) \geq \theta\}$. It is shown that Problem $\left(P_{M L}^{\prime \prime}\right)$ is equivalent to the following program:

$$
\left(P_{M L}^{\prime \prime \prime}\right)\left\{\begin{array}{l}
\text { Maximize } \mu_{1}\left(Z_{1 \alpha}^{F}(x)\right) \text { for } D M_{1} \\
\text { Maximize } \mu_{2}\left(Z_{2 \alpha}^{F}(x)\right) \text { for } D M_{2} \\
\text { subject to } x \in X
\end{array}\right.
$$

where $Z_{l \alpha}^{F}(x)=\left(T_{l}^{*}\left(\theta_{l}\right) d_{l}^{2}+d_{l}^{1}-L^{*}(\alpha) \beta_{l}\right) x$. To find a satisfying solution of Problem $\left(P_{M L}^{\prime \prime \prime}\right)$, we first solve the following problem:

$$
\left(P_{M L}^{i v}\right)\left\{\begin{array}{l}
\text { Maximize } \min \left\{\mu_{1}\left(Z_{1 \alpha}^{F}(x)\right), \mu_{2}\left(Z_{2 \alpha}^{F}(x)\right)\right\} \\
\text { subject to } x \in X
\end{array}\right.
$$

It is an easy matter to see that the above program is equivalent to:

$$
\left(P_{M L}^{v}\right)\left\{\begin{array}{l}
\text { Maximize } v \\
\operatorname{subject~to~}\left(T_{1}^{*}(\theta) d_{1}^{2}+d_{1}^{1}-L^{*}(\alpha) \beta_{1}\right) x \leq \mu_{1}^{*}(v) \\
\left(T_{2}^{*}(\theta) d_{2}^{2}+d_{2}^{1}-L^{*}(\alpha) \beta_{2}\right) x \leq \mu_{2}^{*}(v) \\
x \in X
\end{array}\right.
$$

Let $x^{*}$ the solution of Problem $\left(P_{M L}^{v}\right)$, if the decision maker 1 is satisfied with the membership function values $\mu_{l}\left(Z_{l \alpha}^{F}\left(x^{*}\right)\right), l=1,2$, then $x$ is regarded as a satisfactory solution of Problem $\left(P_{M L}\right)$. Otherwise, the decision maker 1 specifies a minimal satisfactory level $\delta \in(0,1]$ and one solves the mathematical program: 


$$
\begin{aligned}
& \operatorname{Minimize}\left(T_{2}^{*}\left(\theta_{2}\right) d_{2}^{2}+d_{2}^{1}-L^{*}(\alpha) \beta_{2}\right) x \\
& \text { subject to }\left(T_{1}^{*}\left(\theta_{1}\right) d_{1}^{2}+d_{1}^{1}-L^{*}(\alpha) \beta_{1}\right) x \leq \mu_{1}^{*}(\delta) \\
& x \in X
\end{aligned}
$$

A part from this level-set based fractile approach for a multilevel optimization problem under fuzziness and randomness, there is also a possibility-based probability approach.

\subsubsection{Methods for Solving Models in the Category $C_{5}$}

Significant progress has been made on ways for dealing with situations in which randomness and fuzziness are inextricably involved in a multi objective programming framework. These advances have generated more interest for this class of optimization problems. In a theoretical ground several concepts of generalized Pareto optimality have been introduced and properly characterized (see [59] [60]). As far as algorithms are concerned, powerful methods based on tools ranging from simple optimization algorithms to sophisticated ones (like hybrid intelligent algorithm [29]) via meetaheuristics [61] have been devised. The most common methods include:

- methods for generating Pareto optimal solutions,

- interactive methods,

- goal programming methods.

As an example of a method for solving a fuzzy stochastic multi objective program. Consider the following multi objective programming problem:

$$
\left(P_{P O}\right)\left\{\begin{array}{l}
\operatorname{Minimize}\left(\tilde{\overline{\bar{C}}}_{1} x, \cdots, \tilde{\bar{C}}_{h} x\right) \\
\text { subject to } x \in X=\left\{x \in \mathbb{R}^{n} \mid A x \leq b, x \geq 0\right\}
\end{array}\right.
$$

where $x$ is an $n$-dimensional decision vector, $A$ an $m \times n$ matrix, $b$ an $m$-dimensional vector and $\tilde{\bar{C}}_{l}=\left(\tilde{\bar{C}}_{l 1}, \cdots, \tilde{\bar{C}}_{l n}\right) \quad$ where $\quad \tilde{\bar{C}}_{l j}, l=1, \cdots, k$ and $j=1, \cdots, n$ are discrete fuzzy random variables. That is value of $\tilde{\bar{C}}_{l j}$ are triangular fuzzy numbers $\tilde{C}_{l j s j}$, depending on a scenario $s_{l} \in\left\{1, \cdots, S_{l}\right\}$ which occurs with probability $p_{l s l}$. The membership function of $\tilde{C}_{l j s j}$ is:

$$
\mu_{\tilde{C}_{l j s l}}(t)= \begin{cases}\max \left\{1-\frac{d l_{j s l}-t}{\beta_{l j}}, 0\right\} & \text { if } t \leq d_{l j s l} \\ \max \left\{1-\frac{t-d l_{j s l}}{\delta_{l j}}, 0\right\} & \text { if } t>d_{l j s l}\end{cases}
$$

where $d_{l j l}, \beta_{l j}, \delta_{l j}$ are respectively the center value, the left spread value and the right spread value of $\bar{C}_{l j}(\omega)$. Following this pattern, we can define the membership function of the fuzzy random variable $\tilde{\bar{C}}_{l j}$ as:

$$
\mu_{\tilde{\bar{c}}_{i j}(\omega)}(t)= \begin{cases}\max \left\{1-\frac{\bar{d}_{i j}(\omega)-t}{\beta_{i j}}, 0\right\} & \text { if } t \leq \bar{d}_{i j}(\omega) \\ \max \left\{1-\frac{t-\bar{d}_{i j}(\omega)}{\delta_{i j}}, 0\right\} & \text { if } t>\bar{d}_{i j}(\omega)\end{cases}
$$


Making use of the extension principle, we have:

$$
\mu_{\bar{C}_{l s l}(x)}(v)= \begin{cases}\max \left\{1-\frac{d_{l s l} x-v}{\beta_{l} x}, 0\right\} & \text { if } v \leq d_{l s l} x \\ \max \left\{1-\frac{v-d_{l s l} x}{\delta_{l} x}, 0\right\} & \text { if } v>d_{l s l} x\end{cases}
$$

and

$$
\mu_{\tilde{\bar{C}}_{l}(\omega) x}(v)= \begin{cases}\max \left\{1-\frac{\bar{d}_{l}(\omega) x-v}{\beta_{l} x}, 0\right\} & \text { if } v \leq \bar{d}_{l}(\omega) x \\ \max \left\{1-\frac{v-\bar{d}_{l}(\omega) x}{\delta_{l} x}, 0\right\} & \text { if } v>\bar{d}_{l}(\omega) x .\end{cases}
$$

Assume that the decision maker has a fuzzy goal for each of the objective function, say "the objective value should be substantially less or equal than some given value". Denote the membership of this fuzzy goal by $\mu_{\tilde{G}}$. Piecewise linear functions are generally used. Now the degree of possibility that the objective function $\tilde{\bar{C}}_{l} x$ attains the fuzzy goal $\tilde{G}_{l}$ is given by:

$$
\Pi_{\tilde{\bar{C}}_{l} x}\left(\tilde{G}_{l}\right)=\sup _{y} \min \left\{\mu_{\tilde{\bar{C}}_{l} x}(y), \mu_{\tilde{\bar{G}}_{l}}(y)\right\} \text {; }
$$

Or in terms of scenario $s_{l} \in\left\{1, \cdots, S_{l}\right\}$.

$$
\Pi_{\tilde{\bar{C}}_{\mid l s}} x\left(\tilde{G}_{l}\right)=\sup _{y} \min \left\{\mu_{\bar{C}_{l s \varphi} x}(y), \mu_{\bar{G}_{\varphi}}(y)\right\} ; l=1, \cdots, k \text {. }
$$

One may use the expectation operator to convert the original problem into deterministic terms. In this case, one maximizes the expected degree of possibility that each of the original functions involving fuzzy random coefficients attains the fuzzy goals. In this case, the resulting deterministic problem is:

$$
\left(P_{M O}^{\prime}\right)\left\{\begin{array}{c}
\text { Maximize } E\left[\Pi_{\tilde{\bar{C}}_{1} x}\left(\tilde{G}_{1}\right)\right] \\
\vdots \\
\text { Maximize } E\left[\Pi_{\tilde{\bar{C}}_{k} x}\left(\tilde{G}_{k}\right)\right] \\
\text { subject to } x \in X
\end{array}\right.
$$

It is proved that Problem $\left(P_{M O}^{\prime}\right)$ is equivalent to the following multi objective program:

$$
\left(P_{M O}^{\prime \prime}\right)\left\{\begin{array}{l}
\text { Maximize } Z_{1}^{\Pi, E}(x) \\
\vdots \\
\text { Maximize } Z_{k}^{\Pi, E}(x) \\
\text { subject to } x \in X
\end{array}\right.
$$

where

$$
Z_{l}^{\Pi, E}=\frac{\sum_{j=1}^{n}\left(\beta_{l j}-\sum_{s_{l}=1}^{S_{l}} p_{l s l} d_{l j s l}\right) x_{j}+Z_{l}^{0}}{\sum_{j=1}^{n} \beta_{l j} x_{j}-Z_{l}^{1}+Z_{l}^{0}},
$$


and

$$
\begin{aligned}
& Z_{l}^{0}=\max _{s_{j} \in\left\{1, \cdots, S_{l}\right\}} \max _{x \in X} \sum_{j=1}^{n} d_{l j l} x_{j} ; l=1, \cdots, k \\
& Z_{l}^{1}=\max _{s_{j} \in\left\{\left\{, \cdots, s_{l}\right\}\right.} \min _{x \in X} \sum_{j=1}^{l} d_{l j s l} x_{j} ; l=1, \cdots, k .
\end{aligned}
$$

A Pareto optimal solution of Problem $\left(P_{M O}^{\prime \prime}\right)$ may be obtained by using well-known results of deterministic multi objective programming.

\section{Fuzzy Stochastic Optimization Applications}

Fuzzy stochastic optimization is currently applied in a wide variety of domains spanning engineering Economy, Finance, Ecology, Energy, etc. The relevance of FSO was recognized once it became clear that it can be used as a unifying framework for handling situations where fuzziness and randomness co-occur in an optimization setting.

To assert that it is more useful to conceive imprecision in an optimization setting as a variegated whole is not to minimize important research works that have done in Fuzzy Optimization and Stochastic Programming. It is instead to assert that new perspectives for coping with complex real life problems may be gained by integration of both approaches than exclusion. It is virtually impossible to encompass the full spectrum of FSO applications within the scope of a paper. In Table 3, we list some examples of FSO applications in the above mentioned domains.

\section{Concluding Remarks and Suggestions for Future Research}

Fuzzy stochastic system is a fascinating area that allows generating satisfying outputs from fuzzy random inputs subject to a given system restriction. Investigating such systems represents an important research avenue in the discipline of mathematical modeling under uncertainty. Apart from being helpful in several applications, fuzzy stochastic systems can help shed some light on intricate

Table 3. Some applications of FSO.

\begin{tabular}{cc}
\hline Domain & Example of applications \\
\hline Engineering & Planning of solid waste management [62] \\
Economy & Marketing [63] \\
Fiance & Bank hedging decision problems [63] \\
Ecology & Environmental system planning [64] \\
Energy & Electric power planning [65] \\
Water resources & Reservoir strategies [66] \\
Reliability & Time-dependent reliability [67] \\
Network & Shortest path problem [68] \\
Renewal processes & Queuing applications [69]
\end{tabular}


theoretical issues like impacts of infinitesimal changes on inputs of a mathematical model under uncertainty. When systems under scrutiny boil down to mathematical programs, we fall into the realm of fuzzy stochastic optimization, the subject matter of this paper. Fuzzy Stochastic Optimization has triggered, during the last two decades, an explosion of research worldwide both on theoretical issues, algorithmic aspects and concrete applications. It is therefore worth-while to pause once a while and ask ourselves, what we have done, what is done currently and what are the perspectives in this field. This paper has been written in this spirit. Its coverage has been made with a broad target audience in view.

We have highlighted the modeling power of FSO, its broad applicability as well as significant advances made in terms of both the quantity and the quality of results obtained. The variety of related subjects and the current profusion of scientific publications in the field, reflect its vitality and diversity. Some lines for further developments on this fascinating field are briefly outlined below:

1) Despite the fact that most existing approaches for solving FSO problems bow to epistemologically based prescriptive procedures for dealing with problems involving uncertainty, the model validation has been treated mostly from the perspective of efficiency (doing things right). Efforts should be devoted to strike a good balance between efficiency and effectiveness of designed FSO methods. Some indexes, including poss-prod and prod-poss that serve as windows through which one can appraise the desirability of a given action under considered transformations, have been introduced, but more is needed.

2) Decision makers would perhaps be best served if from a deep comparative analysis of good FSO methods, user-friendly Decision Support Systems (DSSs) could be pushed forward. Such (DSSs) would be of great help advise Decision makers with the choice of the most suitable methods for their problems.

3) In some sensitive cases, it would be preferable to have as a solution to a FSO problem, a fuzzy stochastic quantity in a way to reflect the vagueness and the randomness surrounding the problem. So an interesting direction for future research is that of defining and characterizing fuzzy random solutions for a Fuzzy Stochastic Optimization problem. Algorithms for singling out such solutions should also be designed and implemented.

4) Description of high quality case studies is also needed, in order to have more solid arguments in favour of the underniable importance of FSO methods.

We very much hope to see FSO advances so far, so fast that its influence transforms both the theory and practice of mathematical programming under uncertainty.

\section{Conflicts of Interest}

The authors declare no conflicts of interest regarding the publication of this paper.

\section{References}

[1] Dan, H. (2020) Two-Dimensional Topology Optimization of a Horn Antenna. 
Open Journal of Optimization, 9, 39-46. https://doi.org/10.4236/ojop.2020.93004

[2] Luhandjula, M.K. (2006) Fuzzy Stochastic Linear Programming: Survey and Future Research Directions. European Journal of Operations Research, 174, 1353-1367. https://doi.org/10.1016/j.ejor.2005.07.019

[3] Wang, Z. (2017) Landslide Monitoring Point Optimization Deployment Based on Fuzzy Cluster Analysis. Journal of Geoscience and Environment Protection, 5, 118-122. https://doi.org/10.4236/gep.2017.56012

[4] Qiu, W. (2020) There Also Can Be Fuzziness in Quantum States Itself-Breaking through the Framework and the Principle of Quantum Mechanics. Journal of Modern Physics, 11, 952-966. https://doi.org/10.4236/jmp.2020.116059

[5] Dash, J.K., Panda, S. and Panda, G.B. (2020) A New Method to Solve Fuzzy Stochastic Finance Problem. Journal of Economic Studies. https://doi.org/10.1108/JES-10-2020-0521

[6] De la Rosa De, S., Gil, M.A., Gonzalez-Rodriguez, G., Lopez, M.T. and Lubiano, M.A. (2015) Fuzzy Rating Scale-Based Questionnaires and Their Statistical Analysis. IEEE Transactions on Fuzzy Systems, 23, 111-126. https://doi.org/10.1109/TFUZZ.2014.2307895

[7] Liu, J., Beibei, Z., Guohe, H. and Yan, L. (2019) Multi-Stage Stochastic Fuzzy Random Programming for Food Water-Energy Nexus Management under Uncertainties. Resources, Conservation and Recycling, 155, Article ID: 104665. https://doi.org/10.1016/j.resconrec.2019.104665

[8] Zadeh, L.A. (1968) Probability Measures of Fuzzy Events. Journal of Mathematical Analysis and Applications, 23, 421-427. https://doi.org/10.1016/0022-247X(68)90078-4

[9] Dubois, D. and Prade, H. (1980) Fuzzy Sets and Systems: Theory and Application. Academic Press, Cambridge.

[10] Hirota (1981) Concepts of Probabilistic Sets. Fuzzy Sets and Systems, 5, 31-46. https://doi.org/10.1016/0165-0114(81)90032-4

[11] Buckley, J.J. (2003) Uncertain Probabilities I: The Discrete Case. Soft Computing, 7, 500-505. https://doi.org/10.1007/s00500-002-0234-2

[12] Buckley, J.J. (2004) Uncertain Probabilities II: The Continuous Case. Soft Computing, 8, 193-199. https://doi.org/10.1007/s00500-002-0262-y

[13] Puri, M.L. and Ralescu, D.A. (1986) Fuzzy Random Variables. Journal of Mathematical Analysis and Applications, 14, 409-422. https://doi.org/10.1016/0022-247X(86)90093-4

[14] Qiao, Z., Zhang, Y. and Wang, G.Y. (1994) On Fuzzy Random Linear Programming. Fuzzy Sets and Systems, 65, 31-49. https://doi.org/10.1016/0165-0114(94)90245-3

[15] Katagiri, H., Ishiri, H. and Sakawa, M. (2002) Linear Programming with Random Fuzzy Variables Coefficients. Proceedings of 5 th Czech-Japan Seminar on Data Analysis and Decision Making under Uncertainty, Vol. 1, 55-58.

[16] Liu, B. (2003) Expected Value, Operator of Random Fuzzy Variable. International Journal of Uncertainty, Fuzziness and Knowledge-Based Systems, 11, 195-215. https://doi.org/10.1142/S0218488503002016

[17] Walley, P. (1990) Statistical Reasoning with Imprecise Probabilities.

[18] Ferson, S. and Tucker, W.T. (2006) Sensitivity Analysis with Uncertain Numbers. https://doi.org/10.2172/886899 
[19] Luhandjula, M.K. and Gupta, M.M. (1996) On Fuzzy Stochastic Optimization. Fuzzy Sets and Systems, 81, 47-55. https://doi.org/10.1016/0165-0114(95)00240-5

[20] Liu, B. (1999) Uncertain Programming. John Wiley and Son, New York.

[21] Liu, B. (2000) Dependent-Chance in Fuzzy Environments. Fuzzy Sets and Systems, 109, 96-97. https://doi.org/10.1016/S0165-0114(97)00384-9

[22] Mousavi, S., Karamouz, M. and Menhadj, M. (2004) Fuzzy-State Stochastic Dynamic Programming for Reservoir Operation. Journal of Water Resource Planning Management, 130, 460-470. https://doi.org/10.1061/(ASCE)0733-9496(2004)130:6(460)

[23] Liang, R., Gao, J. and Iwamura, K. (2007) Fuzzy Random Dependent-Chance Bilevel Programming with Applications. Proceedings of the 4th International Symposium on Neural Network, Part II, Nanjing, 3-7 June 2007, 6-32.

[24] Gzogala, E. (1983) On Distribution Function Description of Probabilistic Set and Its Application in Decision Making. Fuzzy Sets and Systems, 10, 21-29. https://doi.org/10.1016/S0165-0114(83)80100-6

[25] Luhandjula, M.K. (1983) Linear Programming under Randomness and Fuzziness. Fuzzy Sets and Systems, 10, 45-55. https://doi.org/10.1016/S0165-0114(83)80103-1

[26] Gzogala, E. and Hirota, K. (1986) Probabilistic Sets: Fuzzy Sets and Stochastic Approach to Decision Control and Recognition Process. Verlag, Köln.

[27] Chakraborty, D. (1995) Optimization in Imprecise and Uncertain Environment. $\mathrm{PhD}$ Thesis, IIT, Kharagpur.

[28] Watada, W.J. (2012) Fuzzy Stochastic Optimization. Springer, Berlin. https://doi.org/10.1007/978-1-4419-9560-5

[29] Liu, B. (2002) Theory and Practice of Uncertain Programming. Springer, Berlin. https://doi.org/10.1007/978-3-7908-1781-2

[30] Chakraborty, D. (2002) Redefining Chance-Constrained Programming in a Fuzzy Environment. Fuzzy Sets and Systems, 125, 327-333.

https://doi.org/10.1016/S0165-0114(00)00133-0

[31] Kratschmer, V. (2001) A Unified Approach to Fuzzy Random Variables. Fuzzy Sets and Systems, 123, 1-9. https://doi.org/10.1016/S0165-0114(00)00038-5

[32] Kampempe, J.-D.B. (2013) Sur la prise en compte de l'imprecision dans la la programmation lineaire multicritere. PhD Thesis, University of Kinshasa, Kinshasa.

[33] Luhandjula, M.K. and Joubert, J.W. (2010) On Some Optimization Models in a Fuzzy Stochastic Environment. European Journal of Operational Research, 207, 1433-1441. https://doi.org/10.1016/j.ejor.2010.07.016

[34] Katagiri, H., Mermeri, L.B., Sakawa, M., Kato, K. and Nishihashi, L. (2005) A Possibilistic and Stochastic Programming Approach to Fuzzy Random MST Problems. IEICE Transaction on Information Systems, E88-D, 1912-1919. https://doi.org/10.1093/ietisy/e88-d.8.1912

[35] Arbaiy, N. and Watada, J. (2012) Linear Fractional Programming for Fuzzy Random Possibilistic Programming Problem. 4th International Conference on Computational Intelligence, Modelling and Simulation, Kuantan, 25-27 September 2012, 42. https://doi.org/10.1109/CIMSim.2012.42

[36] Kacprzyck, J. and Fredizzi, M. (1988) Combining Fuzzy Imprecision with Probabilistic Uncertainty in Decision Making. Lecture Notes, Springer-Verlag, Berlin. https://doi.org/10.1007/978-3-642-46644-1

[37] Qiao, Z., Zhang, Y. and Wang, G. (1995) On Fuzzy Random Linear Programming. 
Fuzzy Sets and Systems, 65, 31-49. https://doi.org/10.1016/0165-0114(94)90245-3

[38] Wang, S. and Huang, G.H. (2013) An Interval Parameter Two-Stage Stochastic Fuzzy Program with Type-2 Membership Functions: An Application to Water Resources Management. Stochastic Environmental Research and Risk Assessment, 27, 1493-1506. https://doi.org/10.1007/s00477-013-0685-2

[39] Kelley, D.G. (1981) Some Results on Random Linear Programs. Methods of Operations Research, 40, 351-355.

[40] Dantzig, G.B. (1993) Linear Programming and Extensions. Princeton University Press, Princeton.

[41] Liu, Y., Bai, X. and Hao, F. (2008) A Class of Random Fuzzy Programming and Its Hybrid PSO Algorithm. Lecture Notes in Computer Science, Vol. 5227, 308-315. https://doi.org/10.1007/978-3-540-85984-0 38

[42] Goldberg, D.E. (1989) Genetic Algorithm in Search, Optimization and Machine Learning. Addison-Wesley, Boston.

[43] Glover, F. (1989) Tabu Search-Part I. ORSSA Journal on Computing, 1, 190-206. https://doi.org/10.1287/ijoc.1.3.190

[44] Aiche, F., Abbas, M. and Dubois, D. (2013) Chance-Constrained Programming with Fuzzy Stochastic Co-Efficient. Fuzzy Optimization and Decision Making, 12, 125-152. https://doi.org/10.1007/s10700-012-9151-8

[45] Qiao, Z. and Wang, G. (1993) On Solutions and Distribution Problems of the Linear Programming with Fuzzy Random Variable Coefficients. Fuzzy Sets and Systems, 50, 120-155. https://doi.org/10.1016/0165-0114(93)90492-Z

[46] Iskander, M.G. (2004) A Possibility Programming Approach for Stochastic Fuzzy Multiobjective Linear Fractional Programs. Computers and Mathematics with Applications, 48, 1603-1609. https://doi.org/10.1016/j.camwa.2004.04.035

[47] Liu, B. (2001) Random Fuzzy Variables and Random Fuzzy Programming. Technical Report 2001-66, Report, Dpt. of Mathematical Sciences, Tsinghua University, Beijing.

[48] Liu, Y., Tang, W. and Li, X. (2011) Random Fuzzy Shock Models and Bivariate Random Fuzzy Exponential Distribution. Applied Mathematical Modelling, 35, 2408-2418. https://doi.org/10.1016/j.apm.2010.11.052

[49] Hettich, R. (1979) Semi-Infinite Programming. Springer Verlag, Berlin. https://doi.org/10.1007/BFb0003879

[50] Mohand, C. and Nguyen, H.T. (1997) A Fuzzifying Approach to Stochastic Programming. Operations Research, 34, 73-96. https://doi.org/10.1007/BF03398512

[51] Luhandjula, M.K. (2011) On Fuzzy Random-Valued Optimization. American Journal of Operations Research, 1, 259-267. https://doi.org/10.4236/ajor.2011.14030

[52] Vajda, S. (1972) Probabilistic Programming. Academic Press, Cambridge. https://doi.org/10.1016/B978-0-12-710150-7.50005-9

[53] Kiu, Y.K. and Liu, B. (2002) Random Fuzzy Programming with Chance Measures Defined by Fuzzy Integrals. Mathematical and Computer Modelling, 36, 509-524. https://doi.org/10.1016/S0895-7177(02)00180-2

[54] Negoita, C.V. and Ralescu, D.A. (1976) On Fuzzy Optimization. Kyberneters, 6, 193-195.

[55] Bellman, R.E. and Zadeh, L.A. (1970) Decision-Making in a Fuzzy Environment. Management Sciences, 17, B141-B164. https://doi.org/10.1287/mnsc.17.4.B141

[56] Ganji, A., Khalili, D., Karamouz, M., et al. (2008) A Fuzzy Stochastic Dynamic Nash 
Game Analysis of Policies for Managing Water Allocation in a Reservoir System. Water Resources Management, 22, 51-66. https://doi.org/10.1007/s11269-006-9143-y

[57] Chaves, P. and Kojiri, T. (2007) Stochastic Fuzzy Neural Network: Case Study of Optimal Reservoir Operation. Journal of Water Resources Planning and Management, 133, 509-518. https://doi.org/10.1061/(ASCE)0733-9496(2007)133:6(509)

[58] Sakawa, M., Nishizaki, I. and Katagiri, H. (2011) Fuzzy Multi Objective Programming. Springer, Berlin. https://doi.org/10.1007/978-1-4419-8402-9

[59] Li, J., Xu, J. and Gen, M. (2011) A Class of Multi Objective Linear Programming Model with Fuzzy Random Coefficient. Mathematical and Computer Modelling, 44, 1097-1113. https://doi.org/10.1016/j.mcm.2006.03.013

[60] Adeyefa, A.S. (2010) Multi Objective Programming Problems with Fuzzy Random Coefficients. Advances in Fuzzy Sets and Systems, 7, 1-16.

[61] Hanafi, S. and Ereville, A. (1998) An Effective Tabu Search Approach for the 0-1 Multidimensional Knap-Sack Problem. European Journal of Operational Research, 106, 659-675. https://doi.org/10.1016/S0377-2217(97)00296-8

[62] Li, Y. and Huang, G. (2010) Dual Interval Fuzzy Stochastic Programming Method for Long-Term Planning of Municipal Solid Waste Management. Journal of Computing in Civil Engineering, 24, 188-202. https://doi.org/10.1061/(ASCE)CP.1943-5487.0000025

[63] Weber, K. and Cromme (2004) Decision Support System in Marketing by Means of Fuzzy Stochastic Optimization. GAMM.

[64] Lai, Y.J. and Hwang, C.L. (1993) A Stochastic Possibilistic Programming Model for Bank Hedging Decision Problem. Fuzzy Sets and Systems, 57, 351-363. https://doi.org/10.1016/0165-0114(93)90029-H

[65] Teghem, J. and Kunsch, P. (1985) Multi Objective Decision Making under Uncertainty. An Example of Power Systems. In: Haines and Chankong, Eds., Decision Making with Multiple Objectives, Springer-Verlag, Berlin. https://doi.org/10.1007/978-3-642-46536-9 32

[66] Chaves, P. and Kojiri, T. (2006) Deriving Reservoir Operational Strategies Considering Water Quantity and Quality Objectives by Stochastic Fuzzy Neural Networks. Advances in Water Resources, 30, 1329-1341. https://doi.org/10.1016/j.advwatres.2006.11.011

[67] Modler, B., Beer, M., Graf, W. and Sickert, J.V. (2006) Time-Dependent Reliability of Textile Strengthened. RC Structures under Considerations of Fuzzy Randomness. Computers and Structures, 84, 585-603. https://doi.org/10.1016/j.compstruc.2005.10.006

[68] Ishii, H. and Konno, T. (1998) A Stochastic Inventory Problem with Fuzzy Shortage Cost. European Journal of Operational Research, 106, 90-94. https://doi.org/10.1016/S0377-2217(97)00173-2

[69] Wang, S., Liu, Y.K. and Watada, J. (2009) Fuzzy Random Renewal Process with Queuing Applications. Computers and Mathematics with Applications, 57, 1232-1248. https://doi.org/10.1016/j.camwa.2009.01.030 